\title{
Agricultura De Precisão: Um Mapeamento Da Base Da Scielo
}

\section{Precision Agriculture: A Scielo Base Mapping}

\author{
Ana Clara Cavalcanti de Miranda ${ }^{1}$, Amanda Miranda Veríssimo $^{1}$, Alessandra Carla Ceolin ${ }^{1}$
}

\author{
${ }^{1}$ Universidade Federal Rural de Pernambuco, UFRPE, Brasil \\ Correspondência: Ana Clara Cavalcanti de Miranda. Endereço: R. Manuel de Medeiros, s/n - Dois Irmãos, \\ Recife - PE, 52171-900 E-mail: clara.acmiranda@gmail.com
}

Recebido: 30 de novembro de 2017 Aceito: 15 de dezembro de 2017 Publicado: 31 de dezembro de 2017

DOI: http://dx.doi.org/10.21714/1679-18272017v15Ed.p129-137

\begin{abstract}
Resumo
A agricultura de precisão é um tema altamente relevante, já que o Brasil ocupa um importante lugar no que diz respeito a países exportadores de produtos agrícolas, sendo uma das principais potências agrícolas do mundo. Desta maneira, a agricultura de precisão surge com o objetivo de maximizar a eficiência e eficácia das áreas agrícolas, através de estudos do solo e da variabilidade espacial, utilizando tecnologia de ponta para ajustar as deficiências nas zonas de manejo. Consequentemente, faz-se necessário saber a situação da produção das publicações acadêmicas sobre o tema. Assim sendo, o foco deste artigo tem como base verificar tais publicações acadêmicas sobre a AP e suas abordagens. Para esse fim realizou-se uma pesquisa bibliométrica quantitativa descritiva na base de dados da SciELO, sendo analisados 184 artigos. Tal análise resultou em algumas observações: identificação da inserção em periódicos, ano de publicação, idioma, quantidade de autores, frequência de palavras e/ou termos-chave nos objetivos a fim de relacioná-los às áreas de aplicação e as culturas que mais são abordadas em tais artigos.
\end{abstract}

Palavras-chave: Bibliometria, Abordagem, Agronegócio, Inovação, Agricultura de Precisão.

\begin{abstract}
Precision agriculture is a highly relevant topic since Brazil plays an important role regarding exporters of agricultural products, being one of the main agricultural exporters of the world. Thus, Precision Agriculture emerges with the goal of optimizing efficiency and effectiveness of the agricultural areas through soil studies and spacial variability by using state of the art technology to settle deficiencies in management zones. Accordingly, there is a need for an evaluation of the situation of academic essays about the topic. Therefore, the goal of this study is based on verifying such academic essays on the PA and its approaches. For this purpose, a descriptive quantitative bibliometric research was performed on SciELO data base with 184 analyzed essays. This analysis resulted in: identification of participation in periodicals, publishing year, language, number of authors, keywords or keyterms frequency on the essays objectives in order to relate them to the areas of application and the yield that are most quoted in such essays.
\end{abstract}

Keywords: Bibliometrics, Approach, Agribusiness, Innovation, Precision Agriculture.

Esta obra está licenciada sob uma Licença Creative Commons Attribution 3.0.

\section{Introdução}

As empresas desenvolvem suas atividades buscando antecipar consequências que venha a ser negativas, visando uma melhoria dos resultados. Em tempos de alta tecnologia, procuram adequar-se as necessidades atuais para melhor desenvolver os seus processos. Dentro do agronegócio não é diferente, tecnologia e terras sempre foram aliadas para uma melhor adequação dos recursos na terra gerando resultados cada vez mais voltados a uma boa produção.

A agricultura de precisão (AP) pode ser definida como o processo pelo qual garante-se a uniformização da eficiência em cada metro quadrado da área de manejo, otimizando a produção e diminuindo a variabilidade das áreas da lavoura (PIERCE; NOWAK, 1999). Esse processo se dá através da implantação de ferramentas de sistemas da informação e maquinário adaptado, aliada a adoção de técnicas de tratamento de cada área de acordo 
com as suas deficiências e as suas potencialidades.

Essas técnicas incluem a utilização de mapas por satélite, piloto automático nas colhedoras, fotografias aéreas, amostragem de solo georreferenciada, aplicação em taxa variada de corretivos e fertilizantes, mapas de colheita, entre outras (ANTUNIASSI et al, 2015).

A implantação da AP só foi possível a partir do advento do Sistema por Satélite de Navegação Global (GNSS, sigla em inglês), mais conhecido como GPS (sigla em inglês, Sistema de Posicionamento Global), a partir do ano de 1978 nos Estados Unidos, sendo possível ser acoplado a microprocessadores e realizar o levantamento de dados do solo e do clima. Contudo, o seu uso na agricultura só aconteceu a partir de 1990 com a confecção do "primeiro mapa de produtividade derivado de um monitor de rendimento acoplado ao GPS" (BERNARDI; INAMASU, 2014).

Segundo o Ministério da Agricultura, Pecuária e Abastecimento (2013), a partir deste momento, em 1995, foi iniciada a comercialização de colhedoras com capacidade de mapeamento da produção, gerando uma grande difusão da agricultura de precisão em vários países com produção agrícola, incluindo o Brasil. Foi também a partir desse ano que o assunto adquiriu um viés acadêmico no País com a realização do primeiro simpósio em agricultura de precisão.

Nas últimas décadas verificou-se um avanço vertiginoso da informática e da automação em diversas áreas, incluindo a área agrícola. Essas tecnologias contribuem para uma melhoria das condições de trabalho e promovem a qualidade, a produtividade e a competitividade, além de auxiliarem na preservação ambiental (MINISTÉRIO DA AGRICULTURA, PECUÁRIA E ABASTECIMENTO, 2013, p. 28).

Não obstante, sua aplicação no Brasil ainda era tímida até o final da década de 1990 devido ao não acompanhamento da incorporação de tecnologia de ponta aplicada a produção de máquinas agrícolas pela indústria nacional. Foi só a partir dos anos 2000, que a aplicação da tecnologia à indústria ganhou fôlego, sendo reconhecido como um "salto tecnológico do obsoleto à última geração" (ANFAVEA, 2006, p. 160).

$\mathrm{Na}$ agricultura, os três tipos mais comuns de sistemas de posicionamento são o DGPS (correção via satélite, submétrico), GPS absoluto com correção por algoritmo (também submétrico, mas possui uma degradação da precisão em relação ao tempo, mas funciona muito bem para direcionamento manual) e o RTK ("Real Time Kinematic", milimétrico) (ANTUNIASSI et al, 2015, p. 2).

Além do GPS, a agricultura de precisão faz uso de um software específico, conhecido como Sistema de Informação Geográfica (SIG) e a geoestatística, que delimita a área onde deve haver a intervenção para o melhor manejo da produção.

Intervenção essa que tem como consequência o crescimento da produtividade e a diminuição dos custos na produção já que se sabe exatamente quais produtos, a devida quantidade (aplicação dosada) e o local exato onde devem ser usados. Além de minimizar os danos ao meio ambiente, através da menor contaminação ambiental pelo uso de agrotóxicos, sendo uma forte ferramenta ao que diz respeito a produção sustentável.

Todavia, esses benefícios não são de fáceis mensuração pois levam em conta variáveis difíceis de serem gerenciadas, como as características da lavoura (variabilidade do solo, condições naturais diferenciadas, dentre outros) e os processos adotados por cada produtor. Ou seja, esses benefícios apenas serão percebidos se houver uma variabilidade do solo e dos elementos naturais que tem um impacto sobre a agricultura, além de discrepâncias quanto ao gerenciamento dos processos pelos produtores.

Desta maneira, quanto maior forem esses erros, maior será o retorno econômico percebido. Por exemplo, "se uma propriedade apresenta diferença de produção de duas a dez toneladas por hectare e a aplicação de insumo foi uniforme, então, é fácil entender que em algum ponto está se aplicando excesso de insumo, gerando desperdício e, em outro uma quantidade insuficiente perdendo a oportunidade de se obter uma produção maior" (INAMASU; BERNARDI, 2014).

Por isso, a agricultura de precisão não pode ser tratada como a resolução de todos os problemas da produção agrícola, visto que se não houver grandes variabilidades espaciais e do sistema de produção, sua aplicação poderá ser nula e até apresentar um retorno econômico negativo.

A agricultura de precisão apresenta vasta comprovação de sua eficiência, mas para aquele produtor mais tecnificado e que possua capital suficiente para custear tal 
sistema desde sua implantação, até a colheita dos resultados, tendo capacidade para assumir os riscos com algum possível insucesso na sua instalação (SANTOS, 2014, p. 64).

Diante do exposto, esse trabalho tem como objetivo realizar um estudo bibliométrico, analisando o banco de dados da SciELO sobre agricultura de precisão no período compreendido entre os anos de 2007 a 2017, para a contagem e quantificação dos artigos, visando a construção de indicadores para a identificação de tendências e crescimento do conhecimento acerca da AP, tendo em vista que é um tópico relativamente recente.

Como o uso da agricultura de precisão vem ganhando uma significativa visibilidade no Brasil, é relevante entender como ela é abordada no ambiente acadêmico. Isto posto, esse estudo institui-se como uma importante ferramenta de conhecimento do desenvolvimento de produções acadêmicas a respeito da agricultura de precisão, além de examinar quais assuntos são abordados dentro deste tema central.

\section{Material e Métodos}

A abordagem da pesquisa é qualitativa, cujo o objetivo da amostra é de produzir informações aprofundadas e ilustrativas: seja ela pequena ou grande, o que importa é que ela seja capaz de produzir novas informações (DESLAURIERS; KÉRISIT, 2008, p. 127). A abordagem quantitativa também estruturará o desenvolvimento deste artigo, pois, como afirma Günther (2006), a pesquisa quantitativa permite realizar uma amostra representativa e assegura a possibilidade de uma generalização dos resultados.

A metodologia deste artigo é descritiva e utiliza-se da pesquisa bibliográfica como um método de coleta de dados. Esse tipo de estudo pretende descrever os fatos e fenômenos de determinada realidade (TRIVIÑOS, 1987).

Segundo Gil (1999), as pesquisas descritivas têm como finalidade principal a descrição das características de determinada população ou fenômeno, ou o estabelecimento de relações entre as variáveis.

Quanto ao procedimento, este artigo se enquadra na pesquisa bibliográfica na qual se utiliza fundamentalmente das contribuições de diversos autores. Para Lakatos e Marconi (2001), a pesquisa bibliográfica:

“[...] abrange toda bibliografia já tornada pública em relação ao tema estudado, desde publicações avulsas, boletins, jornais, revistas, livros, pesquisas, monografias, teses, materiais cartográficos, etc. [...] e sua finalidade é colocar o pesquisador em contato direto com tudo o que foi escrito, dito ou filmado sobre determinado assunto [...]" (LAKATOS; MARCONI, 2001, p. 183).

Uma ramificação da análise bibliográfica ocorre através da pesquisa bibliométrica, que por sua vez abrange a abordagem quantitativa desta pesquisa, como diz Lacerda, Ensslin e Ensslin (2012, p.61), o conceito de análise bibliométrica se baseia na evidenciação quantitativa dos parâmetros de um conjunto definido de artigos (portfólio biblioIMAGEM) para a gestão da informação e do conhecimento científico de um dado assunto.

O universo que compreendeu as produções científicas que foram publicadas na base de dados da Scientific Electronic Library Online (SciELO), com o tema indutor "agricultura de precisão".

O SciELO é um programa especial da Fundação de Amparo à Pesquisa do Estado de São Paulo (FAPESP) que proporciona apoio político e financeiro para o desenvolvimento da coleção do SciELO Brasil, interoperando com outras coleções nacionais e temáticas do SciELO, além de garantir a manutenção contínua da plataforma metodológica e tecnológica. (PACKER; COP; LUCCISANO; SPINAK, 2014, p. 16).

Sendo a amostra realizada através dos critérios "apenas artigos", "pesquisas publicadas de 2007 a 2016 " e nos "idiomas em inglês, português e espanhol". Após esse recorte, foram encontrados 186 artigos, sendo essa a amostra da presente pesquisa.

A partir da análise dos artigos, buscou-se identificar, por meio de uma análise da bibliografia, a quantidade de artigos publicados por ano, de autores por artigo, de artigos por idioma e por periódico, bem como a realização de uma análise léxica dos resumos apresentados, a fim de compreender como estão sendo realizados e publicados artigos que tratam desse tema dentro da base de dados da SciELO.

Para Lacerda, Ensslin e Ensslin (2012, p. 61), uma vez determinada a área de conhecimento que será foco da pesquisa, evidencia-se a escolha das palavras-chave que serão utilizadas na busca de referências.

Por fim, buscou-se analisar os objetivos dos artigos e relacionar os termos mais aplicados para compreender 
quais as vertentes das pesquisas realizadas sobre agricultura de precisão.

\section{Resultados e Discussão}

Devido a SciELO ser uma base de dados de pesquisas brasileira, $96,2 \%$ dos artigos analisados é de periódicos do Brasil, com 2,72\% encontrados em periódicos de Portugal, ainda tendo periódicos da Argentina e do Chile com $0,5 \%$ cada.

O SciELO é também usado em muitos países como referência em avaliação de pesquisas, como um complemento das avaliações realizadas com base em índices internacionais. (PACKER; COP; LUCCISANO; SPINAK 2014, p. 18).

Na figura 1 é apresentada a distribuição dos artigos por ano.

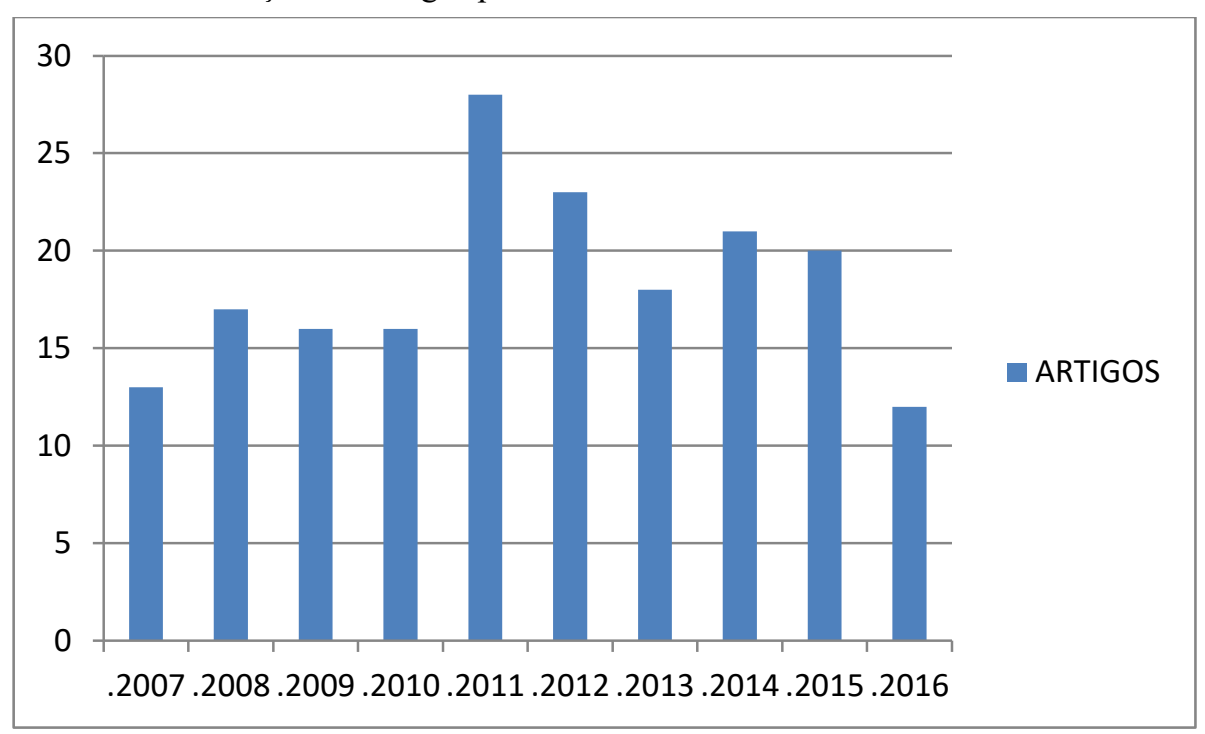

Figura 1: Relação entre anos e quantidade de artigos publicados

Fonte: elaborado pelos autores

A partir de tal observação, infere-se que houve um pico na produção de artigos no ano de 2011, com 28 artigos produzidos, contudo esse número vem sofrendo uma queda desde então. Contudo se comparar este ano de maior produção dentre os 10 últimos anos, com o último ano pesquisado (2016) obtém-se uma diminuição de 16 artigos.

No entanto, a tecnologia é um tema que encontra atualizações constantes e por isso a pesquisa sobre agricultura de precisão, primeiramente por ser um tema atual, como também por tratar diretamente sobre tecnologia não poderia ter suas pesquisas diminuídas. Como afirmam Reetz e Fixen (1999) as tecnologias de agricultura de precisão sempre demandam ferramentas agronômicas de produção mais desenvolvidas.

A tecnologia de AP ainda tem muito a crescer, evidentemente com a resolução dos problemas com que a prática convive atualmente, por exemplo, a falta de mão de obra especializada, altos custos de implantação e falta de compatibilidade entre os aplicativos computacionais e as máquinas e equipamentos (FILHO; CUNHA, 2015, 693).

O fato de ser uma base brasileira de trabalhos científicos e acadêmicos pode ser também a explicação por ter $64 \%$ dos artigos escritos no idioma em português, apesar de haver uma predominância acentuada de artigos produzidos no idioma inglês, $36 \%$, e para concluir essa relação ainda se encontra ainda $1 \%$ de um artigo produzido em espanhol, como se verifica na tabela 1.

Fornecer publicação multilíngue, principalmente nos idiomas inglês, português e espanhol. Isso tem sido crucial para dar aos periódicos latino-americanos a capacidade de alcançar diferentes públicos, especialmente porque os artigos são publicados simultaneamente em duas ou três línguas (PACKER; COP; LUCCISANO; SPINAK 2014, 24). 
Miranda, A. C. C.; Veríssimo, A. M.; Ceolin, A. C. Revista Gestão.Org, v. 15, Edição Especial, 2017. p. 129-137

\section{IDIOMAS E ARTIGOS PUBLICADOS}

\begin{tabular}{ccc}
\hline IDIOMAS & ARTIGOS & \% \\
Português & 118 & 64 \\
Inglês & 66 & 36 \\
Espanhol & 1 & 1 \\
\hline TOTAL & 184 & 100 \\
\hline
\end{tabular}

Tabela 1: Relação entre idiomas e quantidade de artigos publicados

Fonte: elaborado pelos autores

Verifica-se na figura 2 que a maior parte dos artigos foi produzida em conjunto, com inclinação da contribuição entre autores, para haver maiores discussões no tocante ao tema abordado, com a predominância de 54 artigos com 5 autores $(29,34 \%)$.

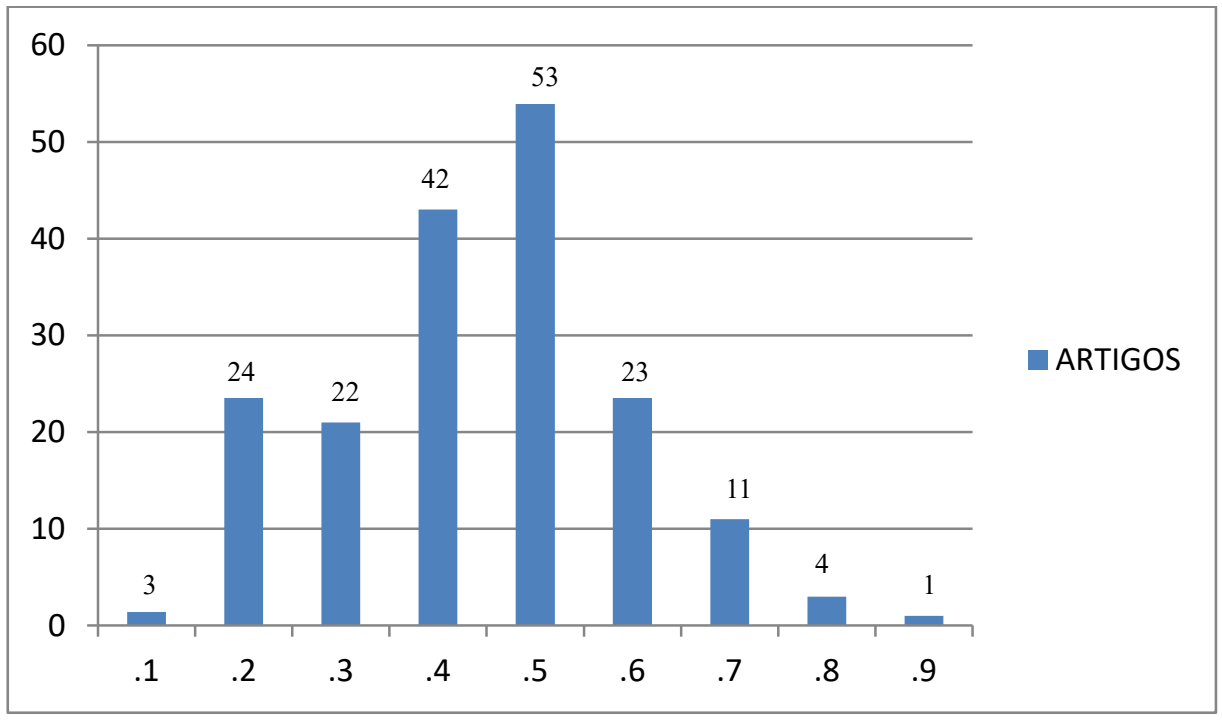

Figura 2: Quantidade de autores por artigos publicados

Fonte: elaborado pelos autores

As áreas temáticas da base da SciELO que estão relacionadas com o tema concentram-se em Ciências Agrárias onde $95 \%$ dos artigos analisados estão agrupados.

Os outros 5\% estão distribuídos entre ciências biológicas, Ciências Exatas e da Terra, Engenharias, Multidisciplinar, Ciências Sociais Aplicadas e Ciências Humanas.

A tabela 2 apresenta a quantidade de artigos publicados por periódicos.

\begin{tabular}{lcc}
\hline \multicolumn{1}{c}{ PERÍODICOS } & ARTIGOS & \% \\
\hline Engenharia Agrícola & 35 & 19,02 \\
Revista Brasileira de Engenharia Agrícola e Ambiental & 25 & 13,59 \\
Revista Brasileira de Ciência do Solo & 18 & 9,78 \\
Ciência Rural & 15 & 8,15 \\
Pesquisa Agropecuária Brasileira & 13 & 7,07 \\
Planta Daninha & 11 & 5,98 \\
Acta Scientiarum. Agronomy & 8 & 4,35 \\
Bragantia (7)/ Ciência e Agrotecnologia (7) & 14 & 7,61 \\
Revista Ceres (6)/ Revista Ciência Agronômica (6) & 12 & 6,52 \\
Revista de Ciências Agrárias & 5 & 2,72
\end{tabular}


Pesquisa Agropecuária Tropical (4)/ Revista Brasileira de Fruticultura (4)

8

4,35

Revista Brasileira de Sementes

Revista Ambiente \& Água

(2)/ Revista Brasileira de Zootecnia

(2)/ Scientia Agricola (2)

Engenharia Sanitaria e Ambiental (1)/ Food Science and Technology (Campinas) (1)/ Horticultura Brasileira(1)/ Idesia (Arica) (1)/ Journal of the Brazilian Chemical Society (1)/ Neotropical Entomology (1)/ Revista Brasileira de Geofísica (1)/ Revista Brasileira de Meteorologia (1)/ Revista Caatinga (1)/ Revista de Ciencia y Tecnología (1)/ Revista de Economia e Sociologia Rural (1)

TOTAL
1,63

6

5,98

11

Tabela 2: Relação entre periódicos e quantidade de artigos publicados

Fonte: elaborado pelos autores

De forma geral, a maioria dos artigos foram publicados em periódicos brasileiros, tendo destaque as publicações realizadas na Revista Engenharia Agrícola, na Revista Brasileira de Engenharia Agrícola e Ambiental, Revista Brasileira de Ciência do Solo, Ciência Rural, estes quatro primeiros periódicos somam $50 \%$ das publicações analisadas. Sendo o periódico que mais pesquisa sobre o tema a Pesquisa de Agropecuária Brasileira. Esse mesmo periódico também foi o que mais obteve trabalhos na análise bibliométrica de Silva et.al (2014), ao pesquisar sobre sensoriamento remoto e agricultura de precisão afirma que os dados indicam que o periódico Engenharia Agrícola teve o maior número de publicações, com 21 artigos totalizados de um total de 109 artigos, entende-se assim que este periódico é um dos mais requisitados quando se trata de agricultura de precisão.

O periódico Engenharia Agrícola iniciou seus trabalhos desde 1972 e possui o caráter de edição o técnicocientifico da SBEA - Associação Brasileira de Engenharia Agrícola. Tendo como classificação B1 em Ciências Agrárias pelo sistema Qualis da Capes. Tendo como missão a publicação de artigos científicos, artigos técnicos e revisões bibliográficas inéditos, fomentando a divulgação do conhecimento prático e científico na área de Engenharia Agrícola.

Dessa forma, percebe-se que não se tem um periódico que esteja relacionado a área de ciências aplicadas que trabalhe diretamente com agricultura de precisão, ficando assim uma lacuna desta área no desenvolvimento de pesquisas sobre o assunto abordado, pois na agricultura de precisão também apresenta um modo de pensar ou abordar o sistema de produção (CERQUEIRA et al., 2009).

A figura 3 apresenta uma nuvem de palavras que foi elaborada a partir das palavras ou termos de maior importância dentro dos objetivos dos artigos analisados. A análise léxica foi realizada com a ajuda do sistema do site wordcounter360, como também com o auxílio do excel. A nuvem representa a quantidade de vezes a qual as palavras ou termos se encontram dentro dos artigos. Lemos (2016) apresenta a nuvem de palavras dando o codinome de nuvem de tags, o qual afirma que é utilizada para poder categorizar de forma visual, títulos, imagens, etiquetas, rótulos entre outros.

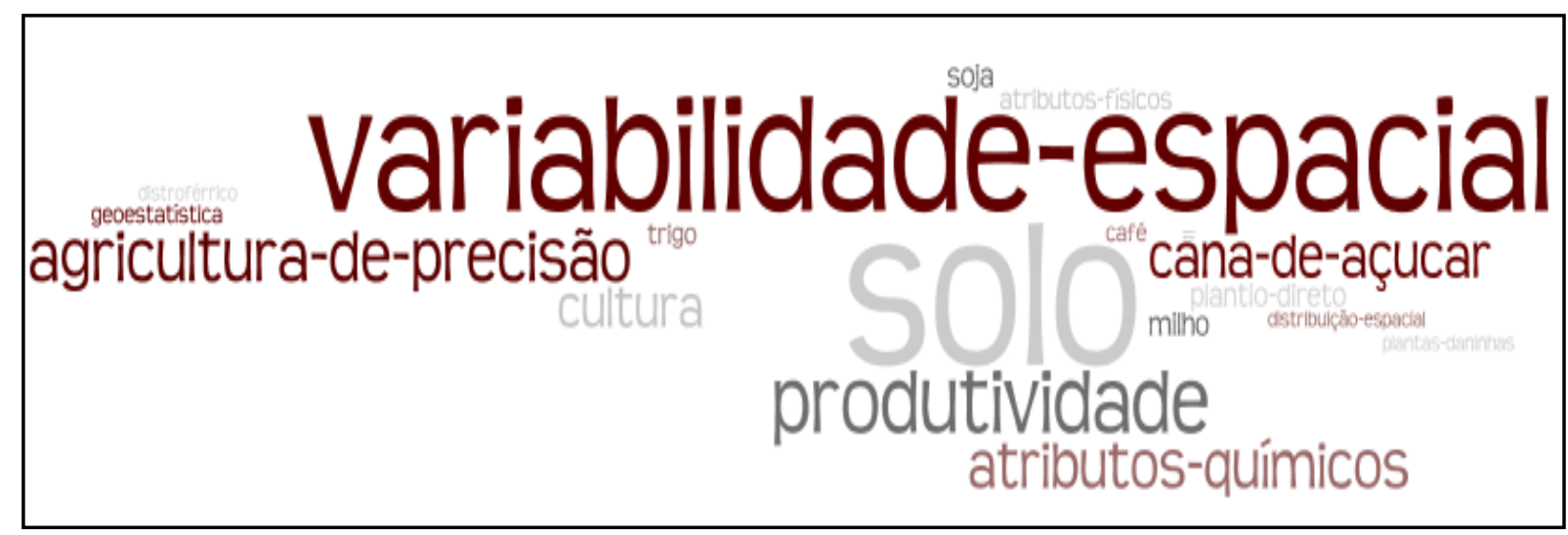

Figura 3: Nuvem das palavras-chave dos artigos pesquisados

Fonte: Elaborado pelos autores a partir do website http://www.wordle.net. 
O procedimento de utilização da nuvem de tags possibilita iniciar uma primeira análise de conteúdo geral... ferramenta que pode auxiliar na categorização da fluidez de conteúdo (LEMOS, 2016, p. 17).

Ressalta-se a ocorrência de mais artigos sobre os plantios de: cana-de-açúcar (14), milho (9), soja (9), trigo (8) e café (7), cujos três primeiros produtos são as principais culturas na agricultura brasileira, segundo o levantamento sistemático da produção agrícola produzido pelo IBGE.

Os termos mais utilizados nesses artigos foram: solo (85), variabilidade espacial (46), produtividade (26), agricultura de precisão (21) e atributos químicos (20).

É com a junção do estudo do "SOLO", palavra que aparece (85) vezes nos objetivos dos artigos analisados, e da "VARIABILIDADE ESPACIAL", (46) vezes, que se surge então o princípio do estudo sobre agricultura de precisão, que aparece (21) vezes, termo chave desta pesquisa. O que ratifica a importância do conhecimento sobre tais fatores na implantação da agricultura de precisão, visto que só havendo variabilidade espacial, há retorno na implantação da AP.

Observando a figura 4, reafirma-se o que foi encontrado na nuvem de palavras.

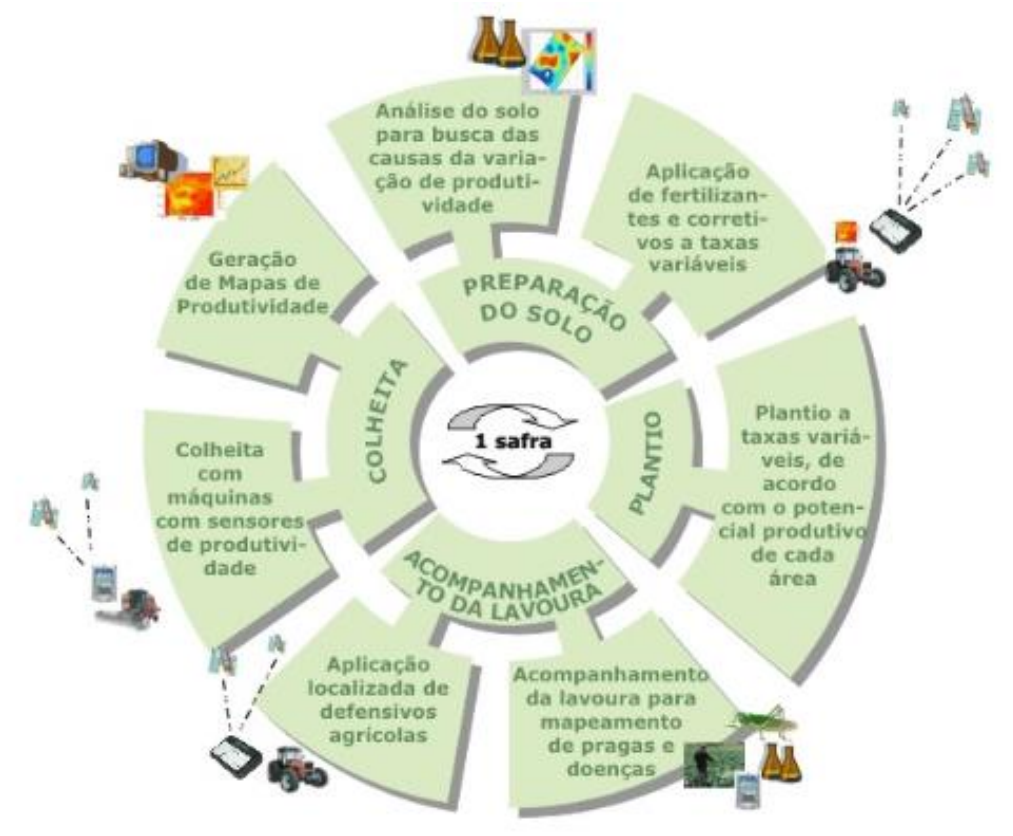

Figura 4: Etapas possíveis para Agricultura de Precisão

Fonte: https://www.agrolink.com.br/georreferenciamento/agricultura-de-precisao_361504.html

A atuação da agricultura de precisão ocorre diretamente com a preparação do solo, com o plantio e a colheita das culturas. Fazendo esse acompanhamento da lavoura através do mapeamento de pragas, através da variabilidade e aplicando adequadamente os atributos químicos segundo sua variabilidade, tudo isto em busca de uma melhor produtividade, como diz Batchelor et al. (1997) manejar a variabilidade da cultura aumenta a produtividade e os lucros.

Inseridas nesta atuação estão três etapas fundamentais para a aplicação da AP, são elas: a coleta de dados, com o objetivo de se conhecer a variabilidade espacial e temporal da cultura; a análise de dados; e a tomada de decisão sobre a melhor técnica a ser utilizada (SANTOS, 2014).

\section{Considerações Finais}

Apesar de haver constância na pesquisa sobre agricultura de precisão na base da SciELO percebe-se que no ano de 2016 houve uma leve queda no número de produções e sabendo que para atuar com a agricultura de precisão, envolve-se a tecnologia e com ela atualizações constantes, por isso a necessidade das pesquisas não diminuírem, sendo assim um convite a comunidade acadêmica a se voltarem para esse tema.

Observa-se também que nos periódicos pesquisados, em sua maioria, se encontram mais direcionados ao tema da agricultura ou até agribusiness, havendo esta lacuna voltada as áreas de ciências aplicadas, que tem a 
possibilidade de pesquisar sobre esse movimento da agricultura de precisão, ajudando diretamente na organização do desenvolvimento aplicado desta tecnologia.

Houve, também, uma avaliação que a maioria dos artigos analisados trata diretamente da variabilidade espacial em seus objetivos, realizando reflexões em cima da melhor produtividade, buscando entender qual cultura adequada para o plantio ou quais atributos químicos ideais para cada ação.

Conclui-se que se faz necessário a compreensão do que se pesquisa sobre o tema como um conhecimento prévio, fazendo um estudo doa assuntos e autores que se abordam para melhor direcionar as pesquisas que estão por vir em cima dos temas tratados e assim fazer um direcionamento adequado.

\section{Referências}

ANFAVEA. Associação nacional dos fabricantes de veículos automotores. Indústria Aumomobilística Brasileira - 50 anos. São Paulo: Autodata editora, 2006. 195 p. Disponível em: $<\mathrm{http}$ ://www.virapagina.com.br/anfavea_50anos/>. Acesso em: 13 mai. 2017.

ANSElmi, A. A. Adoção da Agricultura de Precisão no Rio Grande do Sul. Porto Alegre: Universidade Federal do Rio Grande do Sul, 2012. 105 p. Dissertação, Programa de Pós-Graduação em Agronegócios, Universidade Federal do Rio Grande do Sul, Porto Alegre, 2012. Disponível em: $<$ http://www.lume.ufrgs.br/bitstream/handle/ 10183/40495/000826500.pdf?sequence=1>. Acesso em: 13 mai. 2017.

ANTUNIASSI, U.R.; BAIO, F.H.R; SHARP, T.C. Agricultura de Precisão. In: ABRAPA - Associação Brasileiro dos Produtores de Algodão. (Org.). Algodão no Cerrado do Brasil. $3^{\mathrm{a} e d .}$ Brasília: Eleusio Curvelo Freire, 2015, v., p. 767-806

BATCHELOR, B.; WHIGHAM, K.; DEWITT, J., Precision agriculture: introduction to precision agriculture. Iowa Cooperative Extension, $1997.4 \mathrm{p} . \quad$ Disponível em: $<\mathrm{http}: / /$ www.extension.iastate.edu/Pages/precisionag/prec-ag.pdf>. Disponível em: 18 abr. 2017.

BERNARDI, A. C. de C.; INAMASU, R. Y. Adoção da Agricultura de Precisão no Brasil. In: Agricultura de Precisão: Um Novo Olhar. Brasília: Embrapa, 2014. P. 559-577. Disponível em:

$<$ http://ainfo.cnptia.embrapa.br/digital/bitstream/item/113993/1/Agricultura-de-precisao-2014.pdf $>$. Acesso em: 13 mai.2017.

Brasil. Ministério da Agricultura, Pecuária e Abastecimento. Agricultura de Precisão. Secretaria de Desenvolvimento Agropecuário e Cooperativismo. Brasília: MAPA/ACS, 2013. P.36. Disponível em: $<\mathrm{https}$ ://rhes.ruralhorizon.org/uploads/documents/boletim_tecnico agricultura_de_precisao.pdf $>$. Acesso em: 28 nov. 2017.

CERQueira, E. S. A. QUeIROZ, D. M. PINTO, F. C. A. VAlE, S. M. R. L. SANTO, N. T. Sistema de acompanhamento de atividades para pequenos produtores realizarem o manejo localizado. Engenharia na Agricultura, v.17, p.431-437, 2009GIL, A. C. Métodos e técnicas de pesquisa social. 5.ed. São Paulo: Atlas, 1999.

DESLAURIERS, J. \& KÉRISIT, M. O delineamento de pesquisa qualitativa. In: POUPART, Jean et al. A pesquisa qualitativa: Enfoques epistemológicos e metodológicos. Petrópolis, RJ: Vozes, 2008 (p. 127/153).

ROMEU SOARES FILHO ${ }^{1}$, JOÃO P. A. R. DA CUNHA. Agricultura de precisão: particularidades de sua adoção no sudoeste de Goiás - Brasil. Eng. Agríc., Jaboticabal, v.35, n.4, p.689-698, jul./ago. 2015.

GUNTHER, H. Pesquisa Qualitativa Versus Pesquisa Quantitativa: Esta É a Questão? Psicologia: Teoria e Pesquisa Mai-Ago 2006, Vol. 22 n. 2, pp. 201-210

IBGE, Instituto Brasileiro de Geografia e Estatística. Levantamento sistemático da produção agrícola: Pesquisa mensal de previsão e acompanhamento das safras agrícolas no ano civil. v.30; n.4; p.1-84. Rio de Janeiro: $\quad$ abril. $2017 . \quad$ Disponível em: ftp://ftp.ibge.gov.br/Producao_Agricola/Levantamento_Sistematico_da_Producao_Agricola_[mensal]/Fasciculo/ 1spa_201704.pdf>. Acesso em: 12 mai. 2017.

INAMASU, R. Y; BERNARDI, A. C. de C. Agricultura de Precisão. In: Agricultura de Precisão: Um Novo Olhar. Brasília: $\quad$ Embrapa, $2014.21-33$. $<$ http://ainfo.cnptia.embrapa.br/digital/bitstream/item/113993/1/Agricultura-de-precisao-2014.pdf $>$. Acesso em: 13 mai. 2017. 
LACERDA R. T. O., ENSSLIN L., ENSSLIN S. R. Uma análise bibliométrica da literatura sobre estratégia e avaliação de desempenho. Gest. Prod., São Carlos, v. 19, n. 1, p. 59-78, 2012

LAKATOS, E. M.; MARCONI, M. A. Fundamentos metodologia científica. 4.ed. São Paulo: Atlas, 2001.

LEMOS, L. M. P. Nuvem de tags como ferramenta de análise de conteúdo: uma experiência com as cenas estendidas da telenovela Passione na internet. Revista do Programa de Pós-graduação em Comunicação Universidade Federal de Juiz de Fora / UFJF. vol.10, nº 1,abril, 2016.

PIERCE, Francis J.; NOWAK, Peter. Aspects of Precision Agriculture. Advances in Agronomy, 1999, v.67, p.1-85.

REETZ, H. F. Jr.; FIXEN, P. E. Strategic approach to site-specific systems. Site-specific management guidelines. 1999. Disponível em: <http://www.farmresearch.com/SSMG/ssmg-28/SSMG 28.pdf $>$. Acesso em: 04 mai. 2017.

SANTOS, Lucas B. Viabilidade econômica da implantação de agricultura de precisão na cultura do arroz irrigado em Cachoeira do Sul/RS. Santa Maria: Universidade Federal de Santa Maria, 2014. 72 p. Dissertação, Programa de Pós-Graduação em Agricultura de Precisão, Universidade Federal de Santa Maria, Santa Maria, 2014. Disponível em: < http://w3.ufsm.br/ppgap/images/dissertacoes/2014/Lucas-Bauer-dos-Santos.pdf > Acesso em: 28 nov. 2017.

SILVA, S. F; MENDES, D. F.; FERRARI, J. L.; SOUSA, E. F de; GARCIA, R. F.; SANTOS, A. R. Utilização do Sensoriamento Remoto na Agricultura de Precisão: Uma Análise Bibliométrica, Nucleus, v.11, n.2. out. 2014.

TRIVIÑOS, A. N. S. Introdução... à pesquisa em ciências sociais: a pesquisa qualitativa em educação. São Paulo: Atlas, 1987. 